OPEN ACCESS

Edited by:

Jun Peng,

Fujian University of Traditional Chinese

Medicine, China

Reviewed by:

Ki-Tae Ha,

Pusan National University, South

Korea

Seok-Jae Ko,

Kyung Hee University, South Korea

*Correspondence:

Chang-Gue Son

ckson@dju.kr

${ }^{\dagger}$ These authors have contributed equally to this work

Specialty section: This article was submitted to

Ethnopharmacology,

a section of the journal

Frontiers in Pharmacology

Received: 22 April 2021 Accepted: 16 August 2021 Published: 27 August 2021

Citation:

Kim YH, Kim JY, Kwon O-J, Jung SY, Joung J-Y, Yang CS, Lee J-H, Cho J-H and Son C-G (2021) Efficacy of a Traditional Herbal Formula, BanhaSasim-Tang in Functional Dyspepsia Classified as Excess Pattern. Front. Pharmacol. 12:698887. doi: 10.3389/fphar.2021.698887

\section{Efficacy of a Traditional Herbal Formula, Banha-Sasim-Tang in Functional Dyspepsia Classified as Excess Pattern}

\author{
Yun Hee $\mathrm{Kim}^{1 \dagger}$, Jun Young $\mathrm{Kim}^{2 \dagger}$, O-Jin Kwon ${ }^{3}$, So Young Jung ${ }^{3}$, Jin-Yong Joung ${ }^{2}$, \\ Chang Sop $\mathrm{Yang}^{3}$, Jun-Hwan Lee ${ }^{3}$, Jung-Hyo $\mathrm{Cho}^{2}$ and Chang-Gue Son ${ }^{2 *}$ \\ ${ }^{1}$ Korean Medicine Convergence Research Division, Korea Institute of Oriental Medicine (KIOM), Daejeon, Korea, ${ }^{2}$ Liver and \\ Immunology Research Center, Daejeon Oriental Hospital of Daejeon University, Daejeon, Korea, ${ }^{3}$ Korean Medicine Clinical \\ Research Division, Korea Institute of Oriental Medicine (KIOM), Daejeon, Korea
}

This study evaluated the efficacy and safety of Banha-sasim-tang (BST) in patients with functional dyspepsia (FD). BST (Banxia-xiexin-tang in traditional Chinese medicine and Hange-shashin-to in Kampo medicine) is traditionally prescribed for the treatment of dyspepsia with epigastric stiffness and gastric fullness in China, Japan, and Korea. Patients with FD were randomly administered an oral dose (10 g) of BST syrup or placebo, twice a day for 4 weeks. The primary outcome was the symptom checklist part of the Nepean dyspepsia index (NDI). The secondary outcomes were the quality of life (QoL) part of the NDI, functional dyspepsia-related QoL (FD-QoL), and visual analog scale (VAS). A total of 60 patients with FD were screened, and 50 were randomized into BST group $(n=25)$ and placebo group $(n=25)$. Two patients in the placebo group withdrew before the start of the treatment. Administration of BST syrup resulted in improvement in the symptom-related NDI score in the BST group compared with that in the control group; however, the difference was not significant. BST syrup significantly improved "fullness after eating" index of NDI at follow-up time point (2.88 \pm 2.65 vs $4.78 \pm 2.69, p=0.0081)$. In the total score of the QoL section of the NDI and FD-QoL scales, there was no significant improvement in the BST group compared to that in the placebo group. With regard to improvement in overall FD symptoms, the VAS scale showed improvement in both groups, but the difference was not significant. Interestingly, follow-up investigation showed a significantly beneficial effect of BST on FD symptoms, when compared to placebo. Significant improvement observed in VAS score (39.60 \pm 22.29 vs $52.17 \pm 20.55, p=$ 0.048). This indicated that the effect of BST lasted even after the completion of the medication regimen. Overall, our data suggest that while BST showed no significant improvement in the symptom-related NDI score and the QoL related scores in NDI and FDQoL after 4 weeks of treatment, it effectively improved the VAS score and fullness after eating-related symptoms in the follow-up visit.

Clinical Trial Registration: https://cris.nih.go.kr; Identifier KCT 0002013

Keywords: functional dyspepsia, banha-sasim-tang, investigator-initiated, randomized trial, excess pattern 


\section{INTRODUCTION}

Functional dyspepsia (FD) refers to the presence of gastrointestinal symptoms, such as postprandial fullness, early satiety, epigastric discomfort, bloating, and nausea (Lacy et al., 2013). Although these symptoms are non-life-threatening and FD is not related to an increase in mortality, relapsing-remitting symptoms of FD cause impairment in the quality of life (QoL), and the symptoms cause decreased productivity and activity in the workplace (Mahadeva and Goh, 2006; Talley and Ford, 2015). Therefore, a quarter of the patients with FD choose to consult a physician, thus adding to the excessive health care costs, which exceed several billion dollars annually in the U.S.(Tack et al., 2004; Talley et al., 2006b; Talley et al., 2012). However, the conventional treatment remains unsatisfactory for many patients, and up to $50 \%$ of the patients with FD seek alternative herbal treatments such as STW5, which consists of nine herbs (Lacy et al., 2012; Talley and Ford, 2015).

Banha-sasim-tang (BST, Banxia-xiexin-tang in traditional Chinese medicine [TCM] and Hange-shashin-to in Kampo medicine) is composed of seven herbs as follows: Pinellia ternate (Thunb.) Makino., tuber; Scutellaria baicalensis Georgi., root; Panax ginseng C.A. Mey., root and rhizome; Glycyrrhiza uralensis Fisch., root and rhizome; Ziziphus jujuba Mill., fruit; Zingiber officinale Roscoe., rhizome; Coptis chinensis Franch., rhizome (Naito et al., 2003; Organization, 2007; Zhao et al., 2013). BST is traditionally prescribed in China, Japan, and Korea, as the treatment for dyspepsia with epigastric stiffness and gastric fullness. BST is also used for various gastrointestinal (GI) tract disorders such as chronic gastritis and reflux esophagitis (Xia, 2004; Xu, 2006), diarrhea in patients receiving chemotherapy (Gochi et al., 1995; Mori et al., 1998; Kono et al., 2010). The efficacy of BST in gastrointestinal diseases such as functional dyspepsia (Park et al., 2013; Zhao et al., 2013), chronic atrophic gastritis (Cao et al., 2020) and gastroesophageal reflux disease (Dai et al., 2017) has been evaluated in several studies. However, its efficacy of BST is controversial in FD trials. We speculated that these controversial results were due to drug administration in patients without pattern identification considerations based on individual differences and state. The TCM pattern identification is a system of diagnosis used in TCM. According to TCM pattern diagnosis, individuals may show different symptoms and signs depending on their physical conditions and health status even though they may suffer from the same disease. Different symptoms and signs are classified into several "patterns" (called "Zeung" in Chinese), which have been used as diagnostic tools and for prescribing medicinal herbs and acupoints in TCM (Jiang, 2005; Berle et al., 2010; van der Greef et al., 2010; Zhang et al., 2012).

With regard to FD, herbal formulas such as BST, YukgunjaTang, and Hyangsa-Pyeongwi san (Kim et al., 2014; Kim et al., 2017) are prescribed by Korean medicine doctors depending on the differentiation of the patient's symptoms and signs according to diagnostic patterns in Korea. BST was recorded in the old Chinese medical literature, Shan-han-za-bing-lin (傷寒雜病論). The indication of BST in Shan-han-za-bing-lin is "Epigastric stiffness (心下㾂)” which is similar to the epigastric or upper abdominal fullness (World Health Organization, 2007; Jeon et al.,
2019). In this randomized, blinded, parallel-group trial, we classified FD patients with epigastric stiffness and other related signs as "excess pattern" using a questionnaire (Kim et al., 2010) and specifically evaluated the efficacy and safety of BST syrup for the treatment of FD symptoms.

\section{MATERIALS AND METHODS}

\section{Trial Oversight}

This trial was an investigator-initiated, randomized, blinded, parallel-group trial of BST syrup, manufactured by Jeong-woo Pharmaceutical Company Ltd., (Seoul, Korea) versus placebo for patients with FD in the Daejeon Korean Medicine Hospital in Daejeon, Korea. The study was performed according to the rules, guidelines, and regulations of the Ministry of Food and Drug Safety of the Republic of Korea and was approved by the Institutional Review Board of Daejeon Oriental Medical Center (Authorization no.: djomc-136-01). It was registered in the Clinical Research Information Service of the Republic of Korea (KCT 0002013). From July 15th, 2016 to December 31st, 2016, a total of 50 patients were informed about the trial and provided written consent for participation. All subjects provided written informed consent in accordance with the Declaration of Helsinki.

\section{Participants}

Patients aged between 19 and 75 years with dyspepsia symptoms were eligible for enrolment in the study. Eligible subjects met the ROME III criteria (Drossman and Dumitrascu, 2006) and had one or more of the following symptoms: early satiety, postprandial fullness, and epigastric pain or burning that was not due to structural disease in medical assessment (including upper endoscopy) for more than 3 months and an onset of symptoms at least 6 months prior to study enrollment. Additionally, the included patients had two or more of the following eight moderate symptoms: epigastric pain, discomfort, stuffiness, fullness, burning, postprandial fullness, early satiety, and nausea and a total of at least 6 points in the scoring of the eight symptoms using the following severity scale: 0 , mild; 1 , moderate; 2 and 3, severe. Subsequently, eligible subjects met an excess pattern, as determined using an instrument of pattern identification for FD (Kim et al., 2010). The patient exclusion criteria were a history of gastrointestinal surgery (except appendectomy), the presence of gastrointestinal bleeding, intestinal obstruction, gastrointestinal perforation, colorectal cancer, duodenitis, or stomach cancer. Moreover, patients with severe hepatic dysfunction, congestive heart failure, renal failure, and elevation over the normal range of liver enzymes observed two or more times were excluded. Additional exclusion criteria were current or past use of nonsteroidal anti-inflammatory drugs, corticosteroids, or other investigational study drugs within 30 days before study entry, or pregnancy or lactation in women. All participants fully comprehended the purpose of this trial and the risks involved before participation and submitted their informed consent to participate in the study. Finally, a total of 50 patients with FD were enrolled (25 in the BST group and 25 in the placebo group). 


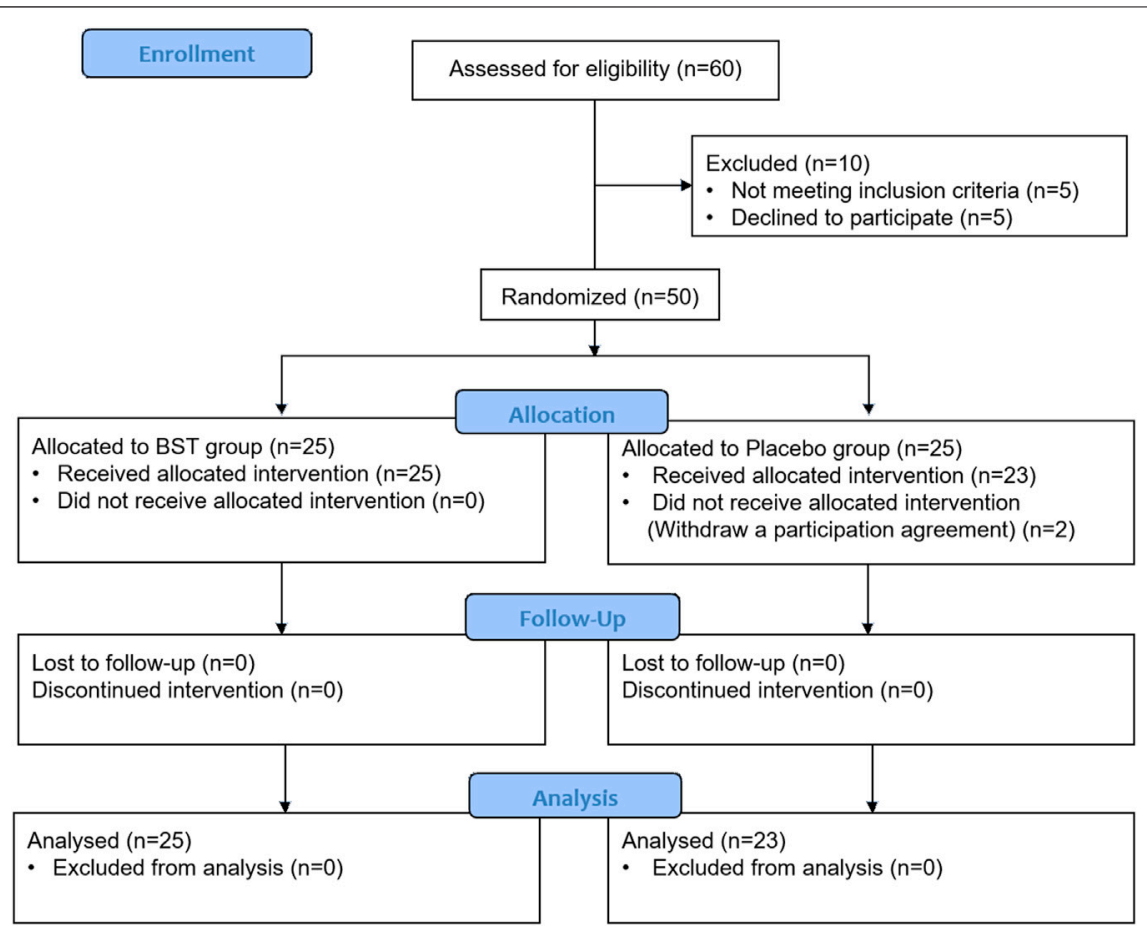

FIGURE 1 | Flow diagram of subject progress through the phase of the RCT.

\section{Study Design}

This study is an investigator-initiated, randomized, blinded, parallel-group trial of BST syrup versus placebo in patients with FD. This clinical trial consisted of 4 weeks of oral administration of BST syrup or placebo, and a 4-weeks follow-up period. Thus, the participants made four visits after the screening. Participants were randomly assigned to two groups: BST syrup or placebo. BST syrup or placebo ( $20 \mathrm{~g}$ each) was administered orally to patients in each group (a single dose was $10 \mathrm{~g}$ ). The random sequence was generated using a computer-generated random number table, and blocked randomization was applied. The investigators, participants, and research sites were blinded to the study group assignment and treatment. Participants were administered BST syrup or placebo twice daily for 4 weeks. After 2 weeks of ingesting BST syrup or placebo, participants visited the clinical center where the leftover BST syrup or placebo was checked to measure compliance. The participants were then given BST syrup or placebo for the remaining 2 weeks. The participants and clinicians were blinded until the end of the study. To evaluate the success of blinding, at the end of the trial, patients were asked about their opinion whether they regarded the drug administered as the active drug or placebo. The whole process of clinical trial is summarized in Figure 1.

\section{Preparation of Drugs and Fingerprinting Analysis}

BST syrup (17JW-08), prepared by Jeong-woo Pharmaceutical Company Ltd. (Seoul, Korea), was produced according to the Korean Good Manufacturing Practice. The BST soft extract was composed of the following herbs: Pinelliae tuber (Pinellia ternate (Thunb.) Makino, $1.178 \mathrm{~g}$ ), Scutellariae radix (Scutellaria baicalensis Georgi, $0.840 \mathrm{~g}$ ), Ginseng radix (Panax ginseng C. A. Mey., $0.803 \mathrm{~g}$ ), Glycyrrhizae radix (Glycyrrhiza uralensis Fisch., $0.732 \mathrm{~g}$ ), Zizyphi fructus (Ziziphus jujuba Mill., $0.512 \mathrm{~g}$ ), Zingiberis rhizoma (Zingiber officinale Roscoe, $0.500 \mathrm{~g}$ ), Coptidis rhizoma (Coptis chinensis Franch., $0.133 \mathrm{~g}$ ), and Zingiberis rhizoma recens (Zingiber officinale Roscoe, $0.077 \mathrm{~g}$ ). All herbal materials were extracted in boiling water, and the extracts were filtered, concentrated, and lyophilized. Following the lyophilized, four additives: $\beta$-Cyclodextrin $(1.500 \mathrm{~g})$, apple concentrate $(1.350 \mathrm{~g})$, carboxymethyl cellulose $(50 \mathrm{mg})$, and sodium benzoate $(5.4 \mathrm{mg})$ with water were added. The matching placebo was provided by the same supplier and was similar in form, flavor, and fragrance to BST syrup, and contained starch $(0.600 \mathrm{~g})$, lactose mixture $(1.412 \mathrm{~g})$, and caramel food coloring (Figure 2A).

To validate the quality of BST syrup, fingerprinting analyses of BST (Figure 2B) and six reference compounds of each herbs (coptisine, baicalein, glycyrrhizic acid, palmatine, berberine, and baicalin) were conducted using high-performance liquid chromatography (HPLC, Agilent 1,100 system, CA, United States), equipped with an auto sampler (G11313A), column oven (GA1316A), binary pump (G1312), diode-arraydetector (DAD), and degasser (GA1379A), as described previously (Jeon et al., 2019).

\section{Assessment of Efficacy}

The effect of BST syrup was evaluated using a symptom-based questionnaire with the Nepean dyspepsia index (NDI) (Talley 


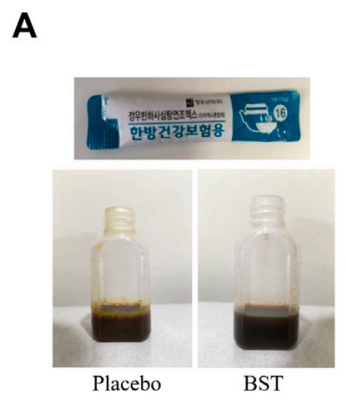

B

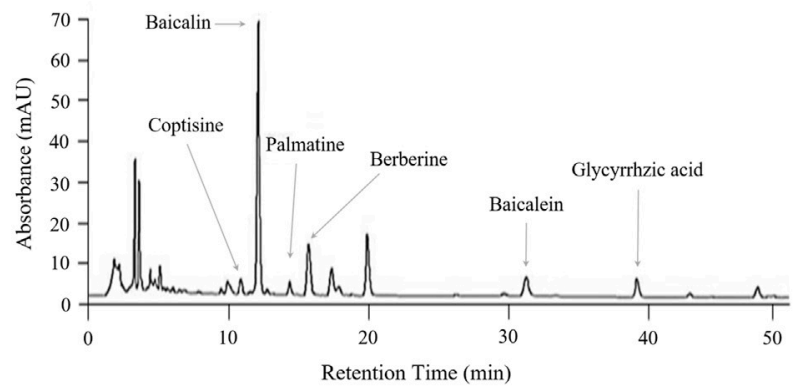

FIGURE 2 | Fingerprint analysis. The real images of placebo and BST syrup (A) and Banha-sasim-tang (BST) using ultra-high-performance liquid chromatographytandem mass spectrometry (UHPLC-MS/MS) (B).

et al., 1999). The NDI consists of two distinct instruments: a symptom checklist and a disease-specific QoL measure. The symptom checklist part of the NDI was used as the primary outcome evaluation. Frequency (scored 0-4), intensity (scored $0-5$ ), and bothersomeness (scored 0-4) were rated according to the state of the symptoms. Higher NDI symptom scores indicated the severity of FD symptoms. Secondary outcome measures consisted of the following items: the total score of QoL section of the NDI, which consists of 25 items; the total score of the validated 21 items of FD-related QoL (FD-QoL) scale [21]; and a change from the baseline in the total score of the visual analog scale (VAS) for measuring severity or pain. We rated each item of the NDI QoL without weighting. We reversely rated the QoL scores so that higher QoL scores account for higher QoL. All outcomes were assessed at days 0,2 , and 4 during BST administration and at 4 weeks after completion of BST administration.

\section{Safety Assessment}

All participants were required to report any adverse events during the trial. Hematological tests for liver and renal functions, vital sign measurements, and electrocardiography were conducted on the second and fourth visits.

\section{Sample Size Calculation}

The purpose of this study was to evaluate the efficacy of BST treatment on FD. In this trial, the sample size was estimated based on the clinical experiences and the results of two previous studies (Lee and Kim, 2013; Kim et al., 2014). The average variation $(\delta)$ was assumed to be 20 and the standard deviation $(\mathrm{SD}=\sigma)$ was assumed to be 22.4 between the two groups. The patients were randomized in a 1:1 ratio to each arm. Results were assessed using a 2-sided test with a statistical power of $80 \%$ and a significance level $(\alpha)$ at $5 \%$. Therefore, this trial included 40 participants, who were divided into two groups of $20(n)$ each, as determined using the following equation:

$$
\mathrm{n}=\frac{2 \times\left(z_{\alpha / 2}+z_{\beta}\right)^{2} \times \sigma^{2}}{\delta^{2}}
$$

Assuming a potential dropout rate of $20 \%, 25$ participants were required to be recruited in each group, totaling 50 participants.

\section{Statistical Analysis}

Statistical analysis was performed using the statistical analysis SAS software (version 9.4, SAS Institute Inc., Cary, NC, United States), and each individual was considered as a unit of analysis. Data were analyzed using intention-to-treat analysis, and all participants who received treatments after randomization were included. All reported $p$-values are two-tailed, and for each analysis, statistical significance was set at $p<0.05$, and data are described as the mean \pm standard deviation (SD). Categorical variables such as baseline clinical characteristics were compared using Fisher's exact test. Continuous variables were compared using ANCOVA test and independent $t$-test for normally distributed data and Wilcoxon rank-sum test for data with non-normal distribution.

\section{RESULTS}

\section{Patient Disposition and Baseline Characteristics}

The trial was performed from July 2016 to December 2016, and a total of 60 candidates were screened. A total of 50 subjects were matched to the inclusion criteria and randomized into two groups, the BST group and the placebo group, at a one-to-one ratio. Two participants in the placebo group dropped out owing to personal reasons before starting the first treatment and were excluded from the modified intention-to-treat (ITT) analysis. Therefore, 23 participants in the placebo group and 25 in the BST group were included in the modified ITT analysis. The baseline characteristics were similar between the two groups, as shown in Table 1.

\section{Efficacy}

BST administration improved the NDI score in the BST group compared to that in the placebo group after 4 weeks; however, the difference was not significant ( $p=0.3102$, Table 2). Nevertheless, BST did not improve the NDI QoL, FD-QoL $(p=0.4358, p=0.0926$, Table 3), and VAS scores ( $p=0.1052$, Table 4).

\section{Follow-Up Study}

To evaluate the long-lasting effect of BST after administration, outcomes were measured at the follow-up visit, that is, 4 weeks 
TABLE 1 | Participants baseline characteristics.

\begin{tabular}{|c|c|c|c|}
\hline & Treatment group $(n=25)$ & Control group $(n=23)$ & $p$-value \\
\hline Gender (Male/Female) ${ }^{a}$ & $8(32.0 \%) / 17$ (68.0\%) & 5 (21.7\%)/18 (78.3\%) & 0.5234 \\
\hline Age $(\text { year })^{b}$ & $50.96 \pm 10.63$ & $49.22 \pm 11.43$ & 0.5947 \\
\hline Height $(\mathrm{cm})^{\mathrm{b}}$ & $162.12 \pm 6.82$ & $159.55 \pm 7.70$ & 0.2357 \\
\hline Body weight $(\mathrm{kg})^{\mathrm{b}}$ & $62.54 \pm 8.40$ & $60.18 \pm 13.21$ & 0.4798 \\
\hline $\mathrm{BMI}(\mathrm{kg} / \mathrm{m} 2)^{\mathrm{b}}$ & $23.78 \pm 2.62$ & $23.45 \pm 3.78$ & 0.7187 \\
\hline Smoking (Yes/No) ${ }^{a}$ & $1(4.0 \%) / 24$ (96.0\%) & $1(4.4 \%) / 22$ (95.6\%) & 0.9999 \\
\hline Drinking (Yes/No) ${ }^{a}$ & $10(40.0 \%) / 15(60.0 \%)$ & $4(17.4 \%) / 19(82.6 \%)$ & 0.1167 \\
\hline Caffeine $\left(\right.$ Yes/No $^{\mathrm{a}}$ & 17 (68.0\%)/8 (32.0\%) & 16 (69.6\%)/7 (30.4\%) & 0.9999 \\
\hline
\end{tabular}

${ }^{a}$ Fisher's exact test.

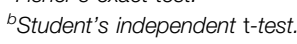

TABLE 2 | Symptom scores using Nepean Dyspepsia Index (NDI).

\begin{tabular}{|c|c|c|c|c|c|}
\hline \multirow[t]{2}{*}{ Items and domains } & \multicolumn{2}{|c|}{ Placebo } & \multicolumn{2}{|c|}{ Banha-sasim-tang (BST) } & \multirow[t]{2}{*}{$p-$ Value $^{a}$} \\
\hline & 0 weeks & 4 weeks & 0 weeks & 4 weeks & \\
\hline \multicolumn{6}{|l|}{ Symptoms } \\
\hline Pain in upper abdomen & $4.69 \pm 3.16$ & $3.22 \pm 2.56$ & $5.32 \pm 3.36$ & $2.68 \pm 2.90$ & 0.4234 \\
\hline Discomfort in upper abdomen & $5.26 \pm 3.0$ & $2.83 \pm 2.48$ & $4.92 \pm 32.7$ & $2.84 \pm 2.97$ & 0.8329 \\
\hline Nausea & $3.60 \pm 2.91$ & $2.09 \pm 3.10$ & $3.56 \pm 3.42$ & $1.04 \pm 1.65$ & 0.1282 \\
\hline Inability to finish a regular meal & $4.95 \pm 3.33$ & $3.09 \pm 3.16$ & $3.44 \pm 2.73$ & $1.96 \pm 1.46$ & 0.1914 \\
\hline Fullness after eating & $6.43 \pm 2.72$ & $4.52 \pm 3.19$ & $6.8 \pm 2.95$ & $3.16 \pm 1.40$ & $0.0247^{\mathrm{a}}$ \\
\hline Pressure in upper abdomen & $6.47 \pm 2.53$ & $3.78 \pm 2.37$ & $5.56 \pm 3.06$ & $3.08 \pm 3.11$ & 0.6543 \\
\hline Burning in upper abdomen & $4.47 \pm 3.69$ & $4.04 \pm 3.30$ & $4.2 \pm 2.73$ & $2.48 \pm 2.83$ & 0.0823 \\
\hline Heart burn & $3.82 \pm 3.21$ & $2.87 \pm 3.09$ & $3 \pm 2.78$ & $2.52 \pm 3.07$ & 0.949 \\
\hline Bitter tasting fluid taste & $4.95 \pm 3.67$ & $3.09 \pm 3.15$ & $3.32 \pm 3.35$ & $2.24 \pm 2.80$ & 0.723 \\
\hline Burping & $6.95 \pm 3.75$ & $4.96 \pm 3.46$ & $5.76 \pm 3.45$ & $3.48 \pm 3.15$ & 0.3082 \\
\hline Cramps in upper abdomen & $2.39 \pm 3.11$ & $1.65 \pm 2.71$ & $1.8 \pm 3.36$ & $1.32 \pm 3.04$ & 0.8914 \\
\hline Chest pain & $3.21 \pm 2.98$ & $2.65 \pm 3.02$ & $3.48 \pm 3.41$ & $1.96 \pm 2.96$ & 0.3186 \\
\hline Vomiting & $1.17 \pm 2.12$ & $1.13 \pm 2.03$ & $1.88 \pm 3.28$ & $0.76 \pm 1.90$ & 0.4945 \\
\hline Bad breath & $2.47 \pm 2.79$ & $1.30 \pm 1.96$ & $1.68 \pm 2.86$ & $0.92 \pm 1.53$ & 0.6667 \\
\hline Bloating in upper abdomen & $6.65 \pm 2.88$ & $3.52 \pm 2.84$ & $6.52 \pm 3.25$ & $3.28 \pm 1.93$ & 0.7544 \\
\hline Total score & $67.57 \pm 26.75$ & $44.74 \pm 31.76$ & $61.24 \pm 25.89$ & $33.72 \pm 26.40$ & 0.3102 \\
\hline
\end{tabular}

${ }^{a} \mathrm{p}$-value by Analysis of covariance (ANCOVA).

TABLE 3 | Quality of Life (QoL) scores using NDI and FD-QoL.

\begin{tabular}{|c|c|c|c|c|c|}
\hline \multirow[t]{2}{*}{ Items and domains } & \multicolumn{2}{|c|}{ Placebo } & \multicolumn{2}{|c|}{ Babha-sasim-tang (BST) } & \multirow[t]{2}{*}{$p$-value ${ }^{a}$} \\
\hline & 0 weeks & 4 weeks & 0 weeks & 4 weeks & \\
\hline \multicolumn{6}{|l|}{ NDI-QOL } \\
\hline Interference & $51.73 \pm 12.49$ & $64.09 \pm 14.20$ & $52.47 \pm 12.26$ & $61.97 \pm 18.01$ & 0.5752 \\
\hline Knowledge/Control & $49.49 \pm 11.43$ & $64.57 \pm 14.63$ & $52.62 \pm 13.36$ & $63.29 \pm 19.89$ & 0.4582 \\
\hline Eat/drink & $57.28 \pm 20.16$ & $68.46 \pm 17.43$ & $60.21 \pm 16.60$ & $67.41 \pm 18.71$ & 0.6057 \\
\hline Sleep disturbance & $59.65 \pm 14.71$ & $73.91 \pm 13.20$ & $65.68 \pm 20.29$ & $70.40 \pm 20.72$ & 0.4063 \\
\hline Total score & $54.54 \pm 12.12$ & $67.76 \pm 11.84$ & $57.75 \pm 11.26$ & $65.77 \pm 17.08$ & 0.4358 \\
\hline \multicolumn{6}{|l|}{ FD-QOL } \\
\hline Eating & $58.48 \pm 18.80$ & $72.83 \pm 17.63$ & $69.00 \pm 16.07$ & $81.00 \pm 12.83$ & 0.2063 \\
\hline Liveliness & $50.27 \pm 17.92$ & $67.93 \pm 19.52$ & $59.00 \pm 18.58$ & $77.00 \pm 13.59$ & 0.1094 \\
\hline Psychological & $63.95 \pm 22.46$ & $80.07 \pm 20.41$ & $75.67 \pm 16.95$ & $87.00 \pm 10.85$ & 0.3447 \\
\hline Role-functioning & $67.93 \pm 18.35$ & $84.06 \pm 14.14$ & $78.83 \pm 15.77$ & $89.00 \pm 10.48$ & 0.3512 \\
\hline Total score & $60.16 \pm 15.79$ & $76.22 \pm 15.05$ & $70.62 \pm 12.52$ & $83.50 \pm 9.74$ & 0.0926 \\
\hline
\end{tabular}

${ }^{a} \mathrm{p}$-value by Analysis of covariance (ANCOVA).

after the final treatment. BST treatment improved the NDI score at follow-up when compared with that observed for the placebo group, with a marginal trend toward significance $(27.76 \pm 24.86$ vs $43.52 \pm 26.01, p=0.0569$, Figure 3A). The score of "fullness after eating" in the NDI index decreased significantly after BST treatment as compared to the placebo group at follow-up $(2.88 \pm$ 
TABLE 4 | Visual Analogue Scale (VAS) scores.

\begin{tabular}{|c|c|c|c|c|c|}
\hline \multirow{2}{*}{$\begin{array}{l}\text { Items and } \\
\text { domains }\end{array}$} & \multicolumn{2}{|c|}{ Placebo } & \multicolumn{2}{|c|}{ BST } & \multirow[t]{2}{*}{$p$-value ${ }^{a}$} \\
\hline & 0 weeks & 4 weeks & 0 weeks & 4 weeks & \\
\hline \multicolumn{6}{|l|}{ VAS } \\
\hline Interference & $62.04 \pm 17.99$ & $45.13 \pm 23.22$ & $64.32 \pm 15.99$ & $34.92 \pm 17.83$ & 0.1052 \\
\hline
\end{tabular}

${ }^{a} \mathrm{p}$-value by Analysis of covariance (ANCOVA).

A

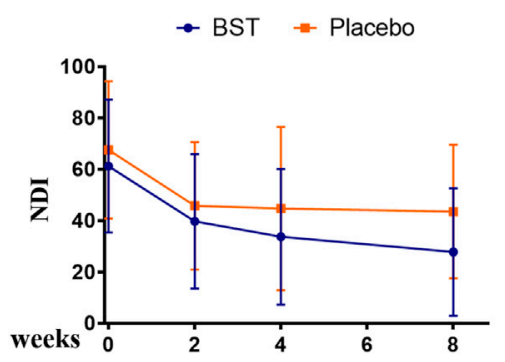

B

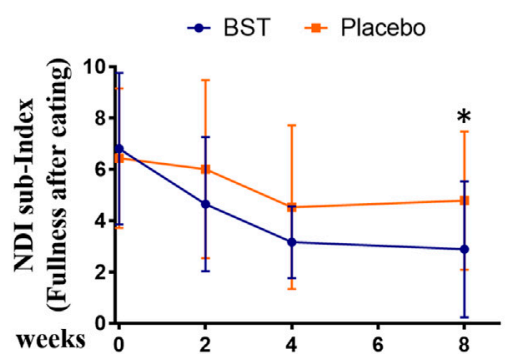

C

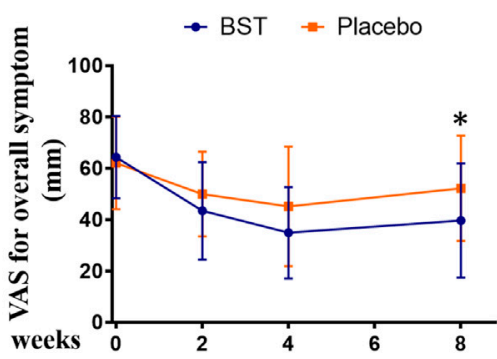

FIGURE 3 | Changes in outcomes between Banha-sasim-tang (BST) and placebo group during the trial. (A) Changes in the Nepean Dyspepsia Index (NDI) symptom score. (B) Changes in the sub-index of NDI symptom score. (C) Changes in the visual analog scale (VAS) for overall symptoms. ${ }^{\star} p<0.05$ vs placebo group.

2.65 vs $4.78 \pm 2.69$, Wilcoxon rank-sum test, $p=0.0081$, Figure 3B). Furthermore, oral administration of BST caused a significant change in VAS compared to that in the placebo group $(39.60 \pm 22.29$ vs $52.17 \pm 20.55, p=0.048$, Figure $3 C)$.

\section{Safety}

No serious adverse events occurred during the trial. Eight cases of mild adverse events were reported in the BST group, which were as follows: nausea (3 cases), headache (1 case), abdominal pain (1 case), epigastric fullness ( 1 case), urticaria (1 case), and acid reflux ( 1 case). Nine cases of mild adverse events, including nausea (2 cases), vomiting (1 case), headache (1 case), abdominal pain (1 case), diarrhea (1 case), dyspepsia (1 case), and slight elevation in aspartate transaminase or alanine aminotransferase levels (2 cases) were reported in the placebo group.

\section{DISCUSSION}

Dyspepsia, defined as pain or discomfort referable to the gastroduodenal region of the gastrointestinal tract, occurs very commonly in the general population. Epidemiological studies have shown that $15-20 \%$ of the population in Western countries has dyspepsia in the course of 1 year (Tack et al., 2004). In studies using upper gastrointestinal endoscopy, more than $70 \%$ of the patients with dyspepsia had FD, while less than $10 \%$ and less than $1 \%$ of the patients with dyspepsia had a peptic ulcer and gastroesophageal cancer, respectively (Ford et al., 2010). Despite the high prevalence of FD, the golden strategy for the treatment of FD remains incomplete, and thus many patients with FD choose other types of treatment. A survey study showed that $36.7 \%$ of the patients with functional gastrointestinal disorders used herbal drugs herbal drugs as an alternative or complementary medicine (Lahner et al., 2013; Ottillinger et al., 2013). BST is a TCM that has been widely used for treating gastrointestinal disease. Both clinical and preclinical studies have shown the beneficial effects of BST for FD (Gan et al., 2014; Jeon et al., 2019).

In this investigator-initiated, randomized, blinded, parallelgroup trial, we aimed to evaluate the efficacy of the herbal drug BST syrup. Results from the primary outcome measurement showed that the mean value of NDI total score was 61.24 in the BST group and 67.57 in the placebo group, and after 4 weeks of treatment, the scores of FD symptoms were ameliorated in both the groups ( $33.72 \pm 26.40$ versus $44.74 \pm 31.76$, respectively). In addition, the score of "fullness after eating" in the NDI index significantly decreased after BST treatment at follow-up. This improvement was consistent with the findings of previous studies that showed that BST improved GI motility and increased gut motility hormone levels (Park et al., 2013; Jeon et al., 2019). As a secondary outcome, we used NDI QoL and FD-QoL to evaluate QoL. Although there are studies with contradictory findings on the relationship between FD and QoL, QoL measurement is an important outcome for disease without biological or clinical markers (Talley et al., 1999; El-Serag and Talley, 2003). In this study, after 4 weeks of treatment, the NDI QoL and FD-QoL scores improved in both the groups, but the difference was not significant. To observe improvement in overall FD symptoms, we used VAS, and the VAS scale showed improvement in both groups, but the difference was not significant. 
Interestingly, follow-up investigation showed a significantly beneficial effect of BST on FD symptoms, when compared to placebo. Significant improvement observed in VAS score. NDI also showed a pattern of improvement, with a considerable trend toward significance. This indicated that the effect of BST lasted even after the completion of the medication regimen. Collectively, our data suggested that BST did not have any significant effects on FD compared to placebo after 4 weeks of treatment. However, BST showed a statistically significant improvement in the followup visit. Considering the prolonged effect of BST in comparison with placebo, this result suggests that BST may not be a palliative treatment but a radical cure on FD. In the safety considerations for both groups, all adverse events were mild and temporary. Moreover, all adverse events, except for urticaria, were related to the prior symptoms of the subjects. The adverse events were, therefore, evaluated as "definitely not related" or "probably not related," and BST syrup or placebo administration was not stopped for any patient.

Taken together, our results suggest that BST may be moderately helpful in FD via improvement of the overall symptoms, even though FD is a heterogeneous disorder and associated with complicated pathophysiological features, (Talley and Ford, 2015; Enck et al., 2017). These improvements may be due to the diverse functions of BST, such as anti-inflammatory function, enhancing gastric motility and protective function of mucosa (Nakazono et al., 2010; Shaofang et al., 2018; Bai et al., 2019; Jeon et al., 2019; Ji et al., 2021; Zenitani et al., 2021).

In TCM and Korea medicine, it is considered best practice to customize herbal formula for each patient. Even though patients are diagnosed with the same disease, they may be prescribed different or modified herbal medicine according to individual differences and disease states. Therefore, the administration of drugs to patients without considerations of individual differences may not be suitable for herbal medicines and thus, do not show significant statistical differences in RCTs (Yuan and Lin, 2000; Flower et al., 2014). In this trial, we performed RCT based on pattern identification by considering disease states and individual differences, and we identified the role of BST in FD. However, this study included a relatively small number of participants. Thus, further studies with diverse populations are needed to evaluate the efficacy of BST. Additionally, the improvement in FD was not assessed via biological evaluation. Although there are no biological markers of $\mathrm{FD}$, previous studies showed that BST

\section{REFERENCES}

Ara, T., Honjo, K., Fujinami, Y., Hattori, T., Imamura, Y., and Wang, P. L. (2010). Preventive Effects of a Kampo Medicine, Orento on Inflammatory Responses in Lipopolysaccharide Treated Human Gingival Fibroblasts. Biol. Pharm. Bull. 33, 611-616. doi:10.2485/jhtb.19.4310.1248/bpb.33.611

Bai, Y., Chen, Y., Chen, Y., Yan, M., Wei, H., Wang, H., et al. (2019). Efficacy of Banxia Xiexin Decoction in a Rat Model of Chronic Atrophic Gastritis. J. Tradit Chin. Med. 39, 867-874.

Berle, C. A., Cobbin, D., Smith, N., and Zaslawski, C. (2010). A Novel Approach to Evaluate Traditional Chinese Medicine Treatment Outcomes Using Pattern Identification. J. Altern. Complement. Med. 16, 357-367. doi:10.1089/ acm.2009.0367 improved GI motility via an increase in the levels of gut regulatory hormones such as somatostatin, motilin, and gastrin (Naito et al., 2002). Therefore, assessing the level of gut regulatory hormones may be an objective indicator for evaluating the efficacy of BST. Moreover, FD is known to be susceptible to placebo effects, and a previous study reported that the placebo response in FD was approximately $30-40 \%$ (Talley et al., 2006a; Musial et al., 2007). Therefore, other objective indicators, such as novel nutrient drink test (Lim et al., 2014), gastric emptying scintigraphy (Hafeez et al., 2018), would be preferable for discriminating between the effects of BST and placebo on $\mathrm{FD}$, in the future studies.

\section{DATA AVAILABILITY STATEMENT}

The raw data supporting the conclusion of this article will be made available by the authors, without undue reservation.

\section{ETHICS STATEMENT}

The studies involving human participants were reviewed and approved by the Institutional Review Board of Daejeon Korean Medicine Hospital of Daejeon University. The patients/ participants provided their written informed consent to participate in this study.

\section{AUTHOR CONTRIBUTIONS}

YK, JK, and C-GS contributed to the conception and design of the study. O-JK performed statistical analyses. YK and JK wrote the first draft of the manuscript. O-JK, SJ, CY, J-HC, J-YJ and J-HL wrote the sections of the manuscript. C-GS supervised the study.

\section{FUNDING}

This study was supported by grants from the Korea Institute of Oriental Medicine (Grant No. K16121) funded by the Korean government (MSIP).

Cao, Y., Zheng, Y., Niu, J., Zhu, C., Yang, D., Rong, F., et al. (2020). Efficacy of Banxia Xiexin Decoction for Chronic Atrophic Gastritis: A Systematic Review and Meta-Analysis. PloS one 15, e0241202. doi:10.1371/ journal.pone.0241202

Dai, Y., Zhang, Y., Li, D., Ye, J., Chen, W., and Hu, L. (2017). Efficacy and Safety of Modified Banxia Xiexin Decoction (Pinellia Decoction for Draining the Heart) for Gastroesophageal Reflux Disease in Adults: a Systematic Review and MetaAnalysis. Evidence-Based Complement. Altern. Med. 2017, 1-17. doi:10.1155/ 2017/9591319

Drossman, D. A., and Dumitrascu, D. L. (2006). Rome III: New Standard for Functional Gastrointestinal Disorders. J. Gastrointestin Liver Dis. 15, 237-241.

El-Serag, H. B., and Talley, N. J. (2003). Health-related Quality of Life in Functional Dyspepsia. Aliment. Pharmacol. Ther. 18, 387-393. doi:10.1046/j.13652036.2003.01706.x 
Enck, P., Azpiroz, F., Boeckxstaens, G., Elsenbruch, S., Feinle-Bisset, C., Holtmann, G., et al. (2017). Functional Dyspepsia. Nat. Rev. Dis. Primers 3, 17081-17120. doi:10.1038/nrdp.2017.81

Flower, A., Witt, C., Liu, J., Ulrich-Merzenich, G., Muir, K., Yu, H., et al. (2014). GP-TCM Unabridged Guidelines for Randomised Controlled Trials Investigating Chinese Herbal Medicine (CHM). Eur. J. Integr. Med. 6, 186-210. doi:10.1016/j.eujim.2013.07.011

Ford, A. C., Marwaha, A., Lim, A., and Moayyedi, P. (2010). What Is the Prevalence of Clinically Significant Endoscopic Findings in Subjects with Dyspepsia? Systematic Review and Meta-Analysis. Clin. Gastroenterol. Hepatol. 8, 830-837. e831-832 doi:10.1016/j.cgh.2010.05.031

Gan, Y., Liu, H., Yang, L., and Yang, K. (2014). Effect of Banxiaxiexin Tang on Treatment of Functional Dyspepsia: a Meta-Analysis of Randomized Controlled Trials. J. Tradit Chin. Med. 34, 140-144. doi:10.1016/s02546272(14)60067-4

Gochi, A., Hirose, S., Sato, K., Hiramatsu, S., Asakura, A., Tokuoka, H., et al. (1995). The Effect of Hange-Shashin-To and Rikkunshi-To against the Digestive Symptoms after Gastrectomy. Jpn. J. Gastroenterol. Surg. Nihon Shokaki Geka Gakkai Zasshi 28, 961-965. doi:10.5833/jjgs.28.961

Hafeez, M., Hussain, F., Salamat, A., and Khan, M. B. (2018). Gastric Emptying Scintigraphy in Postprandial Distress Syndrome. Pak J. Med. Sci. 34, 27-31. doi:10.12669/pjms.341.14137

Jeon, Y. J., Lee, J. S., Cho, Y. R., Lee, S. B., Kim, W. Y., Roh, S. S., et al. (2019). Banhasasim-tang Improves Gastrointestinal Function in Loperamide-Induced Functional Dyspepsia Mouse Model. J. Ethnopharmacol 238, 111834. doi:10.1016/j.jep.2019.111834

Ji, W., Wang, T., Xu, Y., An, R., Liang, K., and Wang, X. (2021). Identifying the Active Compounds and Mechanism of Action of Banxia Xiexin Decoction for Treating Ethanol-Induced Chronic Gastritis Using Network Pharmacology Combined with UPLC-LTQ-Orbitrap MS. Comput. Biol. Chem. 93, 107535. doi:10.1016/j.compbiolchem.2021.107535

Jiang, W. Y. (2005). Therapeutic Wisdom in Traditional Chinese Medicine: a Perspective from Modern Science. Discov. Med. 5, 455-461. doi:10.1016/ j.tips.2005.09.006

Kim, J. B., Shin, J. W., Kang, J. Y., Son, C. G., Kang, W., Lee, H. W., et al. (2014). A Traditional Herbal Formula, Hyangsa-Pyeongwi San (HPS), Improves Quality of Life (QoL) of the Patient with Functional Dyspepsia (FD): Randomized Double-Blinded Controlled Trial. J. Ethnopharmacol 151, 279-286. doi:10.1016/j.jep.2013.10.033

Kim, J., Kim, J., Son, C., Kang, W., and Cho, J. (2010). Development of Instrument of Pattern Identification for Functional Dyspepsia. Korean J. Oriental Physiol. Pathol. 24, 1094-1098.

Kim, J., Park, J.-W., Ko, S.-J., Jeon, S.-H., Kim, J.-W., Yeo, I., et al. (2017). Effects of a Herbal Medicine, Yukgunja-Tang, on Functional Dyspepsia Patients Classified by 3-dimensional Facial Measurement: a Study Protocol for Placebo-Controlled, Double-Blind, Randomized Trial. Evidence-Based Complement. Altern. Med. 2017, 1-8. doi:10.1155/2017/2894507

Kono, T., Satomi, M., Chisato, N., Ebisawa, Y., Suno, M., Asama, T., et al. (2010). Topical Application of Hangeshashinto (TJ-14) in the Treatment of ChemotherapyInduced Oral Mucositis. World J. Oncol. 1, 232-235. doi:10.4021/wjon263w

Lacy, B. E., Talley, N. J., Locke, G. R., Iii, Bouras, E. P., Dibaise, J. K., El-Serag, H. B., et al. (2012). Review Article: Current Treatment Options and Management of Functional Dyspepsia. Aliment. Pharmacol. Ther. 36, 3-15. doi:10.1111/j.13652036.2012.05128.x

Lacy, B. E., Weiser, K. T., Kennedy, A. T., Crowell, M. D., and Talley, N. J. (2013). Functional Dyspepsia: the Economic Impact to Patients. Aliment. Pharmacol. Ther. 38, 170-177. doi:10.1111/apt.12355

Lahner, E., Bellentani, S., Bastiani, R. D., Tosetti, C., Cicala, M., Esposito, G., et al. (2013). A Survey of Pharmacological and Nonpharmacological Treatment of Functional Gastrointestinal Disorders. United Eur. Gastroenterol J 1, 385-393. doi:10.1177/2050640613499567

Lee, A.-R., and Kim, W.-I. (2013). The Retrospective Comparative Study of General Acupuncture Therapy and Hominis Placenta Pharmacopuncture Therapy on Severe Dyspepsia. Korean J. Acupuncture 30, 319-328. doi:10.14406/ acu.2013.041

Lim, C. H., Choi, M. G., Baeg, M. K., Moon, S. J., Kim, J. S., Cho, Y. K., et al. (2014). Applying Novel Nutrient Drink to Clinical Trial of Functional Dyspepsia. J. Neurogastroenterol Motil. 20, 219-227. doi:10.5056/jnm.2014.20.2.219
Mahadeva, S., and Goh, K. L. (2006). Epidemiology of Functional Dyspepsia: a Global Perspective. World J. Gastroenterol. 12, 2661-2666. doi:10.3748/wjg.v12.i17.2661

Mori, K., Hirose, T., Machida, S., and Tominaga, K. (1998). [Kampo Medicines for the Prevention of Irinotecan-Induced Diarrhea in Advanced Non-small Cell Lung Cancer]. Gan To Kagaku Ryoho 25, 1159-1163.

Musial, F., Klosterhalfen, S., and Enck, P. (2007). Placebo Responses in Patients with Gastrointestinal Disorders. World J. Gastroenterol. 13, 3425-3429. doi:10.3748/wjg.v13.i25.3425

Naito, T., Itoh, H., and Takeyama, M. (2003). Comparison of the Effects of HangeShashin-To and Rikkunshi-To on Human Plasma Calcitonin Gene-Related Peptide and Substance P Levels. Biol. Pharm. Bull. 26, 1104-1107. doi:10.1248/ bpb.26.1104

Naito, T., Itoh, H., Yasunaga, F., and Takeyama, M. (2002). Hange-shashin-to Raises Levels of Somatostatin, Motilin, and Gastrin in the Plasma of Healthy Subjects. Biol. Pharm. Bull. 25, 327-331. doi:10.1248/bpb.25.327

Ottillinger, B., Storr, M., Malfertheiner, P., and Allescher, H. D. (2013). STW 5 (Iberogast ${ }^{\circledR}$ )-Aa Safe and Effective Standard in the Treatment of Functional Gastrointestinal Disorders. Wien Med. Wochenschr 163, 65-72. doi:10.1007/ s10354-012-0169-x

Park, J.-W., Ko, S.-J., Han, G., Yeo, I., Ryu, B., and Kim, J. (2013). The Effects ofBanha-Sasim-Tangon Dyspeptic Symptoms and Gastric Motility in Cases of Functional Dyspepsia: A Randomized, Double-Blind, Placebo-Controlled, and Two-Center Trial. Evidence-Based Complement. Altern. Med. 2013, 1-10. doi:10.1155/2013/265035

Shaofang, C., Huiqing, L., Shaodong, C., Yanping, H., Shichuan, W., and Yanxia, H. (2018). Effect of Banxia Xiexin Decoction on Helicobacter Pylori-Related Peptic Ulcers and its Possible Mechanism via the TGF- $\beta /$ Smad Signaling Pathway. J. Traditional Chin. Med. 38, 419-426. doi:10.1016/s0254-6272(18)30633-2

Tack, J., Bisschops, R., and Sarnelli, G. (2004). Pathophysiology and Treatment of Functional Dyspepsia. Gastroenterology 127, 1239-1255. doi:10.1053/ j.gastro.2004.05.030

Talley, N. J., and Ford, A. C. (2015). Functional Dyspepsia. N. Engl. J. Med. 373, 1853-1863. doi:10.1056/NEJMra1501505

Talley, N. J., Haque, M., Wyeth, J. W., Stace, N. H., Tytgat, G. N., Stanghellini, V., et al. (1999). Development of a New Dyspepsia Impact Scale: the Nepean Dyspepsia Index. Aliment. Pharmacol. Ther. 13, 225-235. doi:10.1046/j.13652036.1999.00445.x

Talley, N. J., Locke, G. R., Iii, Herrick, L. M., Silvernail, V. M., Prather, C. M., Lacy, B. E., et al. (2012). Functional Dyspepsia Treatment Trial (FDTT): a DoubleBlind, Randomized, Placebo-Controlled Trial of Antidepressants in Functional Dyspepsia, Evaluating Symptoms, Psychopathology, Pathophysiology and Pharmacogenetics. Contemp. Clin. Trials 33, 523-533. doi:10.1016/ j.cct.2012.02.002

Talley, N. J., Locke, G. R., Lahr, B. D., Zinsmeister, A. R., Cohard-Radice, M., D'elia, T. V., et al. (2006a). Predictors of the Placebo Response in Functional Dyspepsia. Aliment. Pharmacol. Ther. 23, 923-936. doi:10.1111/j.1365-2036.2006.02845.x

Talley, N. J., Locke, G. R., Lahr, B. D., Zinsmeister, A. R., Tougas, G., Ligozio, G., et al. (2006b). Functional Dyspepsia, Delayed Gastric Emptying, and Impaired Quality of Life. Gut. 55, 933-939. doi:10.1136/gut.2005.078634

Van Der Greef, J., Van Wietmarschen, H., Schroën, J., Wang, M., Hankemeier, T., and Xu, G. (2010). Systems Biology-Based Diagnostic Principles as Pillars of the Bridge between Chinese and Western Medicine. Planta Med. 76, 2036-2047. doi: $10.1055 / \mathrm{s}-0030-1250450$

World Health Organization (2007). Regional Office for the Western PacificWHO International Standard Terminologies on Traditional Medicine in the Western pacific Region. Available at: https://apps.who.int/iris/handle/10665/206952.

Xia, J. (2004). Medicinal Herbs Used in Pairs for Treatment of 98 Cases of Chronic Gastritis. J. Tradit Chin. Med. Tsa Chih Ying Wen Pan/sponsored By All-china Assoc. Traditional Chin. Med. Acad. Traditional Chin. Med. 24, 208-209.

Xu, G. (2006). Treatment of Reflux Laryngopharyngitis with Modified Banxia Xiexin Tang (Pinellia Decoction for Draining the Heart)-Aa Report of 40 Cases. J. Tradit Chin. Med. Tsa Chih Ying Wen Pan/sponsored By All-china Assoc. Traditional Chin. Med. Acad. Traditional Chin. Med. 26, 127-131.

Yuan, R., and Lin, Y. (2000). Traditional Chinese Medicine: an Approach to Scientific Proof and Clinical Validation. Pharmacol. Ther. 86, 191-198. doi:10.1016/S0163-7258(00)00039-5

Zenitani, M., Sasaki, T., and Oue, T. (2021). Kampo Medicines Rikkunshito and Hangeshashinto Prevent Cisplatin-Induced Intestinal Mucosal 
Injury in Rats. J. Pediatr. Surg. 56, 1211-1218. doi:10.1016/j.jpedsurg.2021. 03.033

Zhang, A., Sun, H., Wang, P., Han, Y., and Wang, X. (2012). Future Perspectives of Personalized Medicine in Traditional Chinese Medicine: a Systems Biology Approach. Complement. Ther. Med. 20, 93-99. doi:10.1016/j.ctim.2011.10.007 Zhao, L., Zhang, S., Wang, Z., Wang, C., Huang, S., Shen, H., et al. (2013). Efficacy of Modified Ban Xia Xie Xin Decoction on Functional Dyspepsia of Cold and Heat in Complexity Syndrome: a Randomized Controlled Trial. Evidence-Based Complement. Altern. Med. 2013, 1-8. doi:10.1155/2013/812143

Conflict of Interest: The authors declare that the research was conducted in the absence of any commercial or financial relationships that could be construed as a potential conflict of interest.
Publisher's Note: All claims expressed in this article are solely those of the authors and do not necessarily represent those of their affiliated organizations, or those of the publisher, the editors and the reviewers. Any product that may be evaluated in this article, or claim that may be made by its manufacturer, is not guaranteed or endorsed by the publisher.

Copyright (๑) 2021 Kim, Kim, Kwon, Jung, Joung, Yang, Lee, Cho and Son. This is an open-access article distributed under the terms of the Creative Commons Attribution License (CC BY). The use, distribution or reproduction in other forums is permitted, provided the original author(s) and the copyright owner(s) are credited and that the original publication in this journal is cited, in accordance with accepted academic practice. No use, distribution or reproduction is permitted which does not comply with these terms. 